\title{
DESENVOLVIMENTO JUVENIL POSITIVO \\ E PROJETOS DE VIDA: UMA REVISÃO SISTEMÁTICA \\ DA LITERATURA INTERNACIONAL
}

\author{
CHRISTIAN WINTERS* \\ JULIANO PORTO DE CERQUEIRA LEITE** \\ BRUNA CAROLINE PEREIRA*** \\ GABRIELA PAGANO VIEIRA**** \\ LETÍCIA LOVATO DELLAZZANA-ZANON*****
}

\begin{abstract}
RESUMO
O desenvolvimento juvenil positivo se refere a ser o melhor que cada um pode ser e a otimizar desenvolvimento durante a adolescência e o início da vida adulta. Muitos adolescentes desenvolvem suas potencialidades em conjunto com o desenvolvimento de um projeto de vida positivo. Este estudo objetivou realizar uma revisão de literatura internacional dos artigos sobre desenvolvimento juvenil positivo e projetos de vida. Buscou-se identificar como os autores internacionais definem projetos de vida e desenvolvimento juvenil positivo e quais as principais temáticas desses artigos. A busca foi realizada no Portal de Periódicos da CAPES, sendo os descritores utilizados life purpose e positive youth development. Identificaram-se 86 artigos, dos quais se eliminaram 49 por não serem revisados por pares e três por não terem sido publicados durante os anos de 2007 a 2017. Foram lidos os resumos de 34
\end{abstract}

* Bolsista de Iniciação Científica, aluno do curso de Graduação em Psicologia da Pontifícia Universidade Católica de Campinas, christianwinters9@gmail. com .

** Aluno do curso de Graduação em Psicologia da Pontifícia Universidade Católica de Campinas, julianopcleite@gmail.com .

*** Mestranda do Programa de Pós-Graduação em Psicologia da PUC-Campinas. bruna_caroline_2@hotmail.com .

**** Mestranda do Programa de Pós-Graduação em Psicologia da PUC-Campinas.gabrielapaganov@gmail.com .

***** Doutora em Psicologia pela Universidade Federal do Rio Grande do Sul, Professora do Programa de Pós-Graduação em Psicologia da Pontifícia Universidade Católica de Campinas, leticiadellazzana@gmail.com . 
artigos, dos quais 22 foram descartados por não se adequarem à temática do estudo. Analisaram-se 12 artigos. Os resultados indicaram que há uma definição predominante de projetos de vida na literatura internacional. Quanto às principais temáticas dos artigos, observou-se uma predominância de estudos sobre: propriedades psicométricas de escalas, adolescentes em situação de vulnerabilidade, religiosidade adolescente e relações entre projetos de vida e forças de caráter.

Palavras-chave: Projeto de vida; Desenvolvimento juvenil positivo; Adolescência

\section{ABSTRACT}

Positive youth development refers to be the best one can be and to optimize the development during adolescence and the beginning of adulthood. Many adolescents develop their potentials along with the development of a positive purpose in life. This study's objective is to accomplish an international literature's review on articles about purpose in life and positive youth development. It was sought to identify how the international authors define purpose in life and positive youth development and which were the main themes of such articles. The search was done in the Portal de Periódicos CAPES. The key-words were purpose in life and positive youth development. 86 articles were identified, of which 49 were crossed out for not being revised by pairs and 3 for not being published between the years of 2007 and 2017. 34 articles' abstracts were read, of which 22 were removed for not fitting the study's theme. 12 articles were analyzed. The results indicated there is a predominant definition of purpose in life in the international literature. Regarding to the articles' main themes, a predominance of studies was observed about: scales' psychometric properties, adolescents in vulnerable situations, adolescent religiosity and relations between purpose in life and character strengths.

Key-Words: Purpose in life; Positive youth development; Adolescence 


\section{INTRODUÇÃO}

A adolescência tem sido considerada uma fase propícia para a construção de projetos de vida desde os estudos de Piaget (1955/1976;1964/2007) e Erikson (1968/1976). Também na contemporaneidade são vários os autores que compreendem que a adolescência é o período ideal para a construção do projeto de vida. Para Bremm e Bisol (2008) é por meio da elaboração do projeto de vida que o adolescente constrói significados sobre si e sobre o mundo, ressignifica os papéis que ocupava na infância e procura novos referenciais além do grupo familiar. Já na perspectiva de Almeida e Cunha (2003), a adolescência é vista como uma fase de transição, em que o adolescente reconstrói aspectos do passado e elabora projetos de futuro. Para Damon (2009, p. 26) a adolescência é um "período de desenvolvimento transitório, uma espécie de baldeação na estrada rumo a uma autoidentidade madura". Por esse motivo, ele acredita que os adolescentes deveriam gastar algum tempo refletindo sobre si mesmos e sobre seu futuro.

Quanto de projeto de vida, embora seja um tema amplamente abordado desde a década de 1950, não existe um consenso entre os pesquisadores acerca de sua definição (DELLAZZANA-ZANON; FREITAS, 2015). Construir projetos de vida remete à ideia de trazer benefícios para o sujeito (DAMON, 2009; MINEHAN; NEWCOMB; GALAIF, 2000), tais como: (a) conquista da felicidade, da realização e da satisfação pessoal e (b) a garantia de momentos de alegria nos bons tempos e momentos de resiliência nos momentos difíceis. De acordo com Damon (2009), possuir projetos de vida claros pode proteger os jovens de possíveis comportamentos autodestrutivos e os motivar a apresentarem uma atitude positiva perante a vida. Especificamente, ter um projeto de vida pode provavelmente gerar uma série de resultados desejáveis, como um compromisso moral, comportamento pró-social, realização e autoestima elevada (DAMON et al., 2003).

Além disso, não ter um projeto de vida claro pode acarretar efeitos negativos, tanto individuais quanto sociais (DAMON, 1995). Depressão, vícios e uma série de doenças psicossomáticas 
alguns efeitos individuais (DAMON, 1995). Dentre as consequências sociais destaca-se s que a falta de projeto de vida pode resultar em comportamento desviante e destrutivo, improdutividade e incapacidade de sustentar relações interpessoais estáveis (DAMON, 1995). Sendo assim, adolescentes com projetos de vida tendem a se envolver menos com o uso de drogas lícitas ilícitas (MINEHAN; NEWCOMB; GALAIF, 2000). Portanto, em concordância com Mariano e Savage (2009), compreende-se que estudar a temática projetos de vida é fundamental para analisar o progresso do conhecimento científico, tanto em relação ao desenvolvimento do caráter como do bem-estar subjetivo. Conforme enfatizaram Damon et al. (2003), o projeto de vida não é um sonho de adolescente, mas algo que realmente existe e que pode ser investigado.

Recentemente, o projeto de vida tem sido estudado juntamente com o conceito de desenvolvimento juvenil positivo, principalmente na literatura internacional. $O$ juvenil positivo se originou para contrapor-se a uma visão de estudos de psicologia pautada em problemas e dificuldades que os indivíduos apresentam na adolescência (DAMON, 2004). Desde os estudos de Hall (1904) se constata tendência de perceber a adolescência como uma fase tempestuosa do desenvolvimento, repleta de conflitos e problemas. Contudo, investigações indicam que os jovens tendem a escolher caminhos mais positivos do que negativos para suas vidas (BERK, 2009). Assim, além de romper com a ideia de que a adolescência é uma fase problemática do desenvolvimento, a visão do desenvolvimento juvenil positivo fornece a possibilidade de encarar esse período de um modo mais saudável e positivo (DELLAZZANA-ZANON; BACHERT; GOBBO, 2018). Desse modo, pesquisas sobre desenvolvimento juvenil positivo destacam os aspectos positivos do desenvolvimento, considerando os jovens como recursos com potencialidades observáveis (DAMON, 2004; MARIANO; GOING, 2011).

O desenvolvimento juvenil positivo pode ser definido como ser o melhor que se pode ser e a otimização do desenvolvimento durante adolescência e juventude, enfatizando o indivíduo como naturalmente competente e inclinado ao engajamento pró-social 
(DAMON, 2004; MARIANO; GOING, 2011). O desenvolvimento juvenil positivo seria mensurável quando se encontrasse nos jovens cinco componentes (5C): (a) competência, (b) confiança, (c) conexão, (d) caráter e (e) cuidado ou compaixão (ALBERTS et al., 2006). A competência diz respeito a uma visão positiva de ações em diversas áreas, como social, escolar, cognitiva e profissional. A confiança relaciona-se com a presença de autoestima e autoeficácia positivas. A conexão refere-se a relações positivas com pessoas e instituições, como, por exemplo, vínculo do indivíduo com familiares, colegas, escola e comunidade. O caráter está relacionado à existência de um padrão comportamental adequado, à moralidade (noções de certo e errado), ao respeito pelas regras e à honestidade. Finalmente, o cuidado/compaixão faz referência à presença de sentimentos de empatia e simpatia pelos outros (ALBERTS et al., 2006).

Quando os 5C estão presentes surge um sexto, chamado contribuição, o qual é avaliado por meio da participação dos jovens em práticas comunitárias que desempenham um papel ativo na sociedade (LERNER et al., 2005). Assim, que participam de trabalhos voluntários, grupo de jovens e atividades escolares extracurriculares podem ser exemplos da contribuição, pois desempenham atividades para o mundo além do seu "eu". Quando o adolescente acredita que deve contribuir para outrem e quando ele age de acordo com essa crença ocorrem avanços no seu próprio desenvolvimento positivo (LERNER, 2004).

Embora diversas pessoas batalhem para encontrar um projeto de vida no decorrer da idade adulta, constata-se a ênfase desse delineamento no período da juventude. Assim, os jovens passam a questionar pensamentos dominantes, visões de mundo apresentados pelos pais, professores, colegas e demais influências. É durante essa fase que os seres humanos buscam respostas para questões como "com que propósito eu existo?", "por que minha vida importa?" e "qual meu papel no mundo?". Nessa linha de raciocínio, Mariano e Going (2011) defendem que o grau com que os jovens buscam e encontram respostas para essas perguntas tem grandes consequências para a prosperidade deles. 
Resultados de diferentes estudos sobre projeto de vida e desenvolvimento juvenil positivo mostraram que o projeto de vida pode ser considerado um indicador de prosperidade para os jovens (ALBERT et al., 2006; KING et al., 2005). Essas pesquisas revelaram que quando se pergunta aos adolescentes o que significa projeto de vida, observa-se uma gama de respostas referentes a diferentes níveis de engajamento e orientação pró-social (ALBERT et al., 2006; KING et al., 2005). Outros estudos (BRONK, 2005; MARIANO, 2011) destacam que jovens com projetos de vida mais claros contam com variáveis ambientais de figuras modelo, tais como mentores, bons professores, escolas com suporte e apoio de demais indivíduos que vão ao encontro dos interesses dos adolescentes.

Nesse sentido, não há como negar o efeito positivo que o projeto plano de vida exerce na vida dos adolescentes. Mariano e Going (2011) salientam que ter um projeto de vida representa um suporte para enfrentar inúmeras situações adversas que os indivíduos costumam vivenciar durante a adolescência. Da mesma forma, jovens com um projeto de vida bem estruturado apresentam maior vitalidade, energia e abertura a novas ideias do que seus pares sem um projeto. É possível dizer, então, que, para a maioria dos jovens, ter um projeto de vida faz com que eles se sintam bem (MARIANO; GOING, 2011). Isto porque traçar um projeto de vida traz para o jovem uma visão mais clara e conciliatória entre presente e futuro. Sabe-se que muitos adolescentes aprimoram suas potencialidades em conjunto com o desenvolvimento de um projeto de vida positivo (MARIANO; GOING, 2011). Assim, pode-se pensar que o projeto de vida está relacionado com o desenvolvimento positivo do adolescente na medida em que atinge um lugar central na identidade e no funcionamento psicológico do indivíduo (MARIANO; GOING, 2011).

Oportuno destacar algumas considerações: (a) a adolescência é um período do desenvolvimento profícuo para a construção de projeto de vida, (b) adolescentes que têm projetos planos de vida são mais felizes e menos propensos a se envolverem em comportamentos de risco típicos da adolescência, (c) o projeto de vida pode ser entendido como um indicador de prosperidade 
(desenvolvimento juvenil positivo) para muitos adolescentes que desenvolvem suas potencialidades em conjunto com o desenvolvimento planejamento de um desenvolvimento juvenil positivo. Em face de tais argumentações enfatiza-se que este estudo teve por objetivo realizar uma revisão sistemática da literatura internacional sobre desenvolvimento juvenil positivo e projetos de vida. Especificamente, buscou-se identificar e analisar: (a) a definição de desenvolvimento juvenil positivo e projeto de vida utilizada pelos pesquisadores interessados nessa temática, (b) identificar os objetivos dos estudos empíricos internacionais sobre projetos de vida e desenvolvimento juvenil positivo, e (c) identificar os instrumentos utilizados para acessar projetos de vida nos estudos internacionais selecionados.

\section{MÉTODO}

A busca dos artigos foi realizada no Portal de Periódicos da CAPES em outubro de 2017, utilizando-se os seguintes descritores: (a) "life purpose" e (b) "positive youth development". Os descritores poderiam estar presentes no título, nas palavras-chave, no resumo ou no corpo do texto. Os critérios de inclusão para o presente artigo foram: (a) estudos disponíveis online na íntegra, (b) ser revisado por pares, (c) pesquisa com participantes adolescentes e (d) publicados entre 2007 e 2017. Foram excluídos os artigos teóricos ou de revisão de literatura. Trabalharam juntos três pesquisadores, os quais avaliaram separadamente os artigos.

Dos 86 artigos identificados, 49 foram eliminados por não serem revisados por pares e três por não terem sido publicados durante os anos de 2007 a 2017. Foram lidos os resumos de 34 artigos, dos quais 22 foram resultaram descartados por não se adequarem à temática do estudo. Sendo assim, analisaram-se 12 artigos (Figura1). 
Figura 1. Fluxograma do processo de revisão sistemática (Winters, Leite, Pereira, Vieira \& Dellazzana-Zanon).

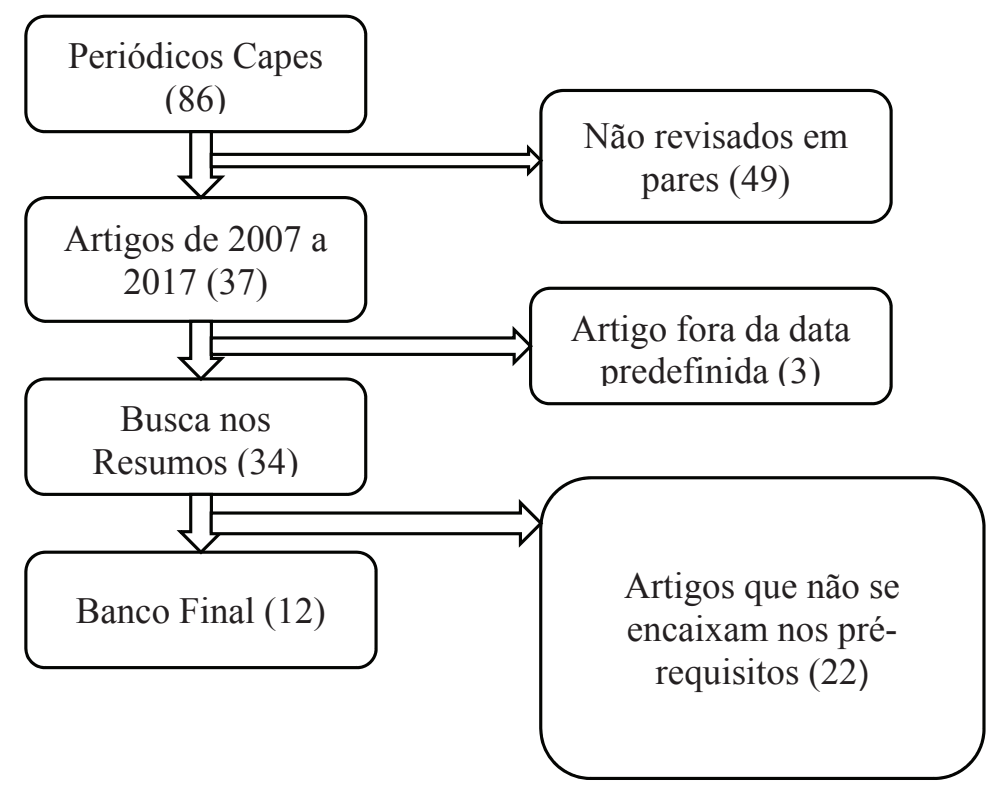

\section{RESULTADOS E DISCUSSÃO}

Todos os 12 artigos selecionados foram publicados no período de 2011 a 2017, sendo nove deles (CHAN; TSAI; FULIGNI, 2015; CHUA; MILFONT, 2014; DAVIS III; KIANG, 2016; MACHELL; DISABATO; KASHDAN, 2016; MALIN; LIAUW; DAMON, 2017; RAMEY et. al., 2015; SHOSHANI; RUSSO-NETZER, 2017; SINK; BULTSMA, 2014; STODDARD; PIERCE, 2015) nos últimos quatro anos, sugerindo o crescente e recente interesse pela temática. Observou-se a média de três publicações por ano, considerando o período analisado (2014 a 2017). Tendo-se em vista os objetivos do estudo, organizou -se os resultados de acordo com os seguintes temas: (a) definição[5] de projeto de vida e de desenvolvimento juvenil positivo, ou seja, informações sobre a apresentação ou não de uma definição de projeto de vida e desenvolvimento juvenil po- 
sitivo; (b) objetivos dos estudos selecionados, e (c) instrumentos para avaliar projeto de vida.

\section{DEFINIÇÃO DE PROJETO DE VIDA}

Dentre os 12 artigos analisados seis apresentaram uma definição de projeto de vida, sendo que a proposta por Damon et al (2003) foi a mais utilizada, estando presente na maioria (5) dos artigos (BLATTNER et al., 2013; LAW, 2012; MALIN; LIAUW; DAMON, 2017; MACHELL; DISABATO; KASHDAN, 2016; PIZZOLATO; BROWN; KANNY, 2011). Para Damon et al (2003, p. 121), projeto de vida seria “uma intenção estável e generalizada de realizar algo que seja ao mesmo tempo significativo para o eu e de consequência para o mundo além do eu". Já na visão de Stoddard e Pierce (2015) projeto de vida é entendido da seguinte forma: metas abrangentes que têm significado pessoal (GEORGE; PARK, 2013) e que fornecem uma estrutura para essas metas e ações de nível inferior e motivam um indivíduo a alocar recursos pessoais para sua realização (MCKNIGHT; KASHDAN, 2009).

Assim, é perceptível que apesar de não existir uma definição comum a todos os pesquisadores sobre projeto de vida, a maior parte da literatura converge na definição proposta por Damon et al. (2003). Dito de outra forma, mesmo não sendo unânime, essa definição segue como a mais utilizada pela literatura internacional sobre a temática (BLATTNER et al., 2013; LAW, 2012; MALIN et al., 2017; MACHELL et al., 2016; PIZZOLATO et al., 2011).

Esses resultados estão em consonância com os encontrados em outro estudo de revisão de literatura (DELLAZZANA-ZANON; FREITAS, 2015). Nessa pesquisa, realizou-se uma revisão de literatura de artigos sobre projetos de vida na adolescência produzidos de 2000 até 2012 com o objetivo de investigar se pesquisadores interessados no tema apresentam ou não uma definição de projeto de vida e, em caso afirmativo, quais são. Os resultados indicaram que todos os artigos internacionais do estudo adotaram a definição que Damon et al. (2003).

Além disso, observou-se que metade dos artigos da amostra não apresentou uma definição de projeto de vida. Esse resultado 
também vai na direção do encontrado no estudo de Dellazzana-Zanon e Freitas (2015), no qual mais de 60\% dos estudos $(63,6 \%)$ não apresentam uma definição explícita de projeto de vida. Esses resultados chamam atenção para dois aspectos: (a) a dificuldade de definir o que são projetos de vida, um tema tão importante para o desenvolvimento do adolescente e (b) muitos estudos, internacionais inclusive, não apresentam as definições operacionais das variáveis com as quais estão trabalhando.

O primeiro objetivo desse artigo era investigar como os estudos internacionais definem projeto de vida e desenvolvimento juvenil positivo. No que se refere às definições de desenvolvimento juvenil positivo, nenhum dos 12 artigos da amostra apresentou uma definição do construto.

\section{OBJETIVO DOS ESTUDOS}

Quanto aos objetivos dos estudos selecionados, observou-se grande variedade, o que demonstra que a investigação das temáticas projetos de vida e desenvolvimento juvenil positivo pode ser feita por meio da associação com diferentes constructos, na medida em que é muito abrangente. Para facilitar a análise no presente estudo os artigos encontrados foram agrupados em quatro temáticas, considerando-se a similaridade de seus objetivos: (a) estudos de propriedades psicométricas de escalas e testes, (b) estudos sobre adolescentes em situação de vulnerabilidade, (c) estudos sobre religiosidade adolescente e (d) um estudo sobre projeto de vida e forças de caráter.

A primeira temática refere-se a estudos com o objetivo de testar propriedades psicométricas ou de validação de escalas e testes psicológicos, incluindo quatro artigos (LAW, 2012; RAMEY et. al., 2015; SHOSHANI; RUSSO-NETZER, 2017; SINK; BULTSMA, 2014). O trabalho de Law (2012) objetivou a validação Existence Subscale of the Purpose in Life Questionnaire, realizada com uma amostra de 2.842 adolescentes com idade entre 11 e 14 anos. Já a pesquisa de Sink e Bultsma (2014) buscou examinar as propriedades psicométricas do Life Perpectives Inventory na versão inglês, com 1.003 adolescentes participantes do estudo. Ramey et al. (2015) tinham por objetivo criar um modelo de mensurar 
o engajamento psicológico e utilizaram uma amostra de 2.117 jovens com faixa etária entre 9 a 24 anos. Por fim, o objetivo do artigo de Shoshani e Russo-Netzer (2017) era a validação da Meaning in Life in Children Questionnaire, que foi aplicada em 1.957 estudantes, com idade entre 9 e 12 anos.

A segunda temática encontrada agrupa três artigos que estudam adolescentes em situação adversas e de vulnerabilidade (CHUA; MILFONT, 2014; MACHELL et al., 2016; PIZZOLATO et al., 2011). Pizzolato et al. (2011) realizaram uma intervenção em um grupo de 209 estudantes de baixo nível econômico, com vistas a promover a obtenção do sucesso acadêmico e o desenvolvimento do projeto de vida. Chua e Milfont (2014) realizaram uma pesquisa longitudinal com 1.774 participantes com idade entre 10 e 15 anos, na qual buscaram analisar a relação entre orientação futura, mecanismos de enfrentamento e estado psicológico. A investigação de Machell et al. (2016) visou verificar se projeto de vida poderia ser um fator de resiliência para uma amostra de 1.256 adolescentes que vivem na pobreza, observando se este constructo tem algum impacto em comportamentos antissociais ou pró-socias.

A terceira temática relaciona dois artigos que têm por objetivo estudar a religiosidade (CHAN et al., 2014; DAVIS III; KIANG, 2016). Assim, Chan et al. (2014) realizaram um estudo longitudinal com 584 adolescentes com vistas a explorar mudanças na afiliação religiosa e identitária, além de relacionar religiosidade com significado e projeto de vida. O estudo de Davis III e Kiang (2016) buscou compreender o desenvolvimento religioso e suas implicações, tendo como participantes 180 adolescentes asiático-americanos.

Três artigos apresentaram temáticas muito distintas das demais, de modo que não foram colocados em nenhuma categoria e serão abordados a seguir (BLATTNER et al., 2013; MALIN et al., 2017; STODDARD; PIERCE, 2015). O estudo de Blattner et al. (2013) buscou examinar a associação entre a busca de um projeto de vida e a autoestima de garotas adolescentes, investigando qual seria o papel de seus pais nessa vivência. O trabalho de Stoddard e Pierce (2015) contou com 196 adolescentes e teve 
por objetivo examinar as relações entre contribuição para comunidade, esperança, projeto de vida e expectativas futuras. Por fim, Malin et al. (2017) visaram descrever de maneira qualitativa quão cedo 1.003 participantes adolescentes apresentaram projetos de vida e em que ações eles mostram, além de conhecer possíveis relações entre projetos de vida e forças do caráter.

\section{INSTRUMENTOS DE PROJETO DE VIDA}

Em relação ao delineamento de pesquisa, a maioria dos artigos (10) é quantitativa, sendo apenas dois de natureza qualitativa. Os instrumentos mais utilizados foram o The Satisfaction with Life Scale (LAW, 2012; SHOSHANI; RUSSO-NETZER, 2017), o The Rosenberg's Test (BLATTNER et al., 2013; DAVIS III; KIANG, 2016) e o Meaning in Life Questionnaire (DAVIS III; KIANG, 2016; SHOSHANI; RUSSO-NETZER, 2017), todos presentes em dois estudos cada. Dentre os artigos, Stoddard e Pierce (2015) foram os que mais utilizaram instrumentos (5), aplicando escalas de quatro ou três itens para investigar os construtos expectativas futuras, esperança, projeto de vida e contribuição da comunidade e também utilizaram o instrumento Neighborhood Collective Efficacy Scale.

Nos 12 artigos da amostra, foram aplicados um total de 31 instrumentos, mantendo-se uma média de 2,5 instrumentos por estudo. Apesar desse número, apenas seis instrumentos foram usados para avaliar projeto de vida: o The Revised Purpose in Life Test (PIL-R), Existential Scale of the Purpose in Life, The Revised $n$ Youth Purpose Survey, The Prosocial Youth Purpose Scale, Youth Purpose Interview Protocole a escala de três itens de Stoddard e Pierce (2015). Nota-se, assim, que nos estudos sobre desenvolvimento juvenil positivo e projetos de vida, muitas outras variáveis foram investigadas.

\section{CONSIDERAÇÕES FINAIS}

O objetivo do presente artigo foi realizar uma revisão sistemática da literatura internacional sobre desenvolvimento juvenil positivo e projeto de vida. Observou-se que o número de os estudos envolvendo essas duas temáticas aumentou ao longo dos últimos anos e que, apesar disso, ainda há lacunas na 
literatura quanto à definição desses dois constructos. No entanto, existe uma predominância pelo uso da definição de Damon et al. (2003) quanto à definição de projetos de vida, o que já havia sido constatado em outra investigação nacional sobre a temática. Por outro lado, não foram encontradas definições sobre desenvolvimento juvenil positivo nos estudos, o que chama atenção.

O tema mais investigado nos estudos da amostra sobre desenvolvimento juvenil positivo e projetos de vida foi propriedades psicométricas de escalas e testes, o que reflete o interesse dos pesquisadores internacionais no processo de construção de instrumentos de avaliação psicológica. Quanto aos aspectos metodológicos, observou-se que os estudos da amostra utilizam prioritariamente delineamentos quantitativos para investigar desenvolvimento juvenil positivo e projetos de vida, o que, por um lado, salienta o interesse dos pesquisadores internacionais por investigações com grandes amostras, mas, por outro, uma carência em pesquisas qualitativas. No que se refere aos instrumentos, todos os estudos trabalhos fizeram uso de um ou mais instrumentos, porém, poucos foram usados para investigar projetos de vida de forma específica.

No que diz respeito às limitações do estudo, deve-se mencionar que, por se tratar de uma revisão de literatura em inglês sobre uma temática ainda pouco estudada no contexto nacional, houve certa dificuldade em elencar os artigos e definir quais eram, de fato, aqueles que tratavam de forma específica dos dois temas de interesse deste estudo: desenvolvimento juvenil positivo e projetos de vida. Além disso, dar conta do objetivo de encontrar definições explícitas desses constructos também revelou-se uma tarefa complexa, pois, em muitas situações os autores dos artigos não deixam isso claro, o que dificulta a compreensão e a análise de quem se propõe a analisar os artigos. Mesmo assim, espera-se que esta revisão chame atenção para a importância de se estudar as interfaces entre essas duas temáticas para a compreensão saudável da adolescência: o desenvolvimento juvenil positivo e a construção do projeto de vida. 


\section{REFERÊNCIAS}

ALBERTS, A. E. et al. Qualitative and quantitative assessments of thriving and contribution in early adolescence: Findings from the 4-H study of positive youth development. Journal of Youth Development, v. 1, n. 2, 2006. Available from: < file:///C:/Users/mirian/Desktop/art.1.pdf>. Cited: 21 aug. 2018. ALMEIDA, A. M. O.; CUNHA, G. G. Representações Sociais do Desenvolvimento Humano. Psicologia: Reflexão e Crítica, v. 16, n. 1, p. 147-155, 2003. BERK, L. E. Child development Boston. 8th. Illinois: Pearson, 2009.

BREMM, E. S.; BISOL, C. A. Sinalizando a adolescência: narrativas de adolescentes surdos. Psicologia Ciência e Profissão, v. 28, n. 2, p. 272-287, 2008. BRONK, K. C. Portraits of purpose: a study examining the ways a sense of purpose contributes to positive youth development, (Doctoral dissertation, Stanford University, 2006). UMI ProQuest Digital Dissertations, AAT 3187267, 2005.

CHAN, M.; TSAI, K.M.; FULLIGNI, A.J. J. Changes in Religiosity Across the Transition to Young Adulthood. Youth Adolescence. v. 44, p. $1555-$ 1566,2015

CHUA, L.W.; MILFRONT, T.L.; JOSE, P.E. J. Coping Skills Help Explain How Future-Oriented Adolescents Accrue Greater Well-Being Over Time. Youth Adolescence, v. 44, p. 2028-2041, 2015.

DAMON, W. Greater expectations: overcoming the culture of indulgence in our homes and schools. New York: Free Press, 1995.

DAMON, W. O que o jovem quer da vida? Como pais e professores podem orientar e motivar os adolescentes. (J. Valpassos, Trans.) São Paulo: Summus, 2009.

DAMON, W.; MENON, J.; BRONK, K. C. The development of purpose during adolescence. Applied Developmental Science, v. 7, n. 3, p. 119-128, 2003. Available from: <http://search-ebscohost-com.ez128.periodicos.capes.gov.br/login.aspx?direct $=$ true $\& \mathrm{db}=$ aph\&AN=10130791\&lang= pt-br\&site=ehost-live. $>$. Cited: 21 aug. 2018.

DAMON, W. What is positive youth development? The Annals of the American Academy of Political and Social Science, n. 591, p. 13-23, 2004. Available from: < http://faculty.wiu.edu/P-Schlag/articles/What $\% 20$ is $\% 20$ Positive\%20Youth\%20Development.pdf.>. Cited: 21 aug. 2018.

DAVIS III, R.F.; KIANG, L. J. Religious Identity, Religious Participation, and Psychological Well-Being in Asian American Adolescents. Youth Adolescence, v. 45, p. 532-546, 2016.

DELLAZZANA-ZANON, L. L.; FREITAS, L. B. L. Uma revisão de literatura sobre a definição de projeto de vida na adolescência. Interação em Psicologia, v. 19, n. 2, p. 281-292, 2015.

DELLAZZANA-ZANON, L. L.; BACHERT, C. M. D.; GOBBO, J. Projetos de vida do adolescente: implicações para a escolarização positiva. In: NAKANO, 
T. C. (Org.). Psicologia positiva aplicada à educação. São Paulo: Vetor Editora, 2018. p. 41-62.

ERIKSON, E. H. Identidade, juventude e crise. (A. Cabral, Trans.). Rio de Janeiro: Zahar. 1976.

HALL, G. Adolescence. New York: Appleton, 1904.

KING, P. E. et al. Thriving in adolescence: the voices of youth-serving practitioners, parents, and early and late adolescents. Journal of Early Adolescence, v. 25, n. 1, p. 94-112, 2005. Available from: <http://journals.sagepub.com. ez128.periodicos.capes.gov.br/doi/pdf/10.1177/0272431604272459>. Cited: 21 aug. 2018.

LERNER, R. M. Liberty: thriving and civic engagement among american youth. Thousand Oaks, CA: Sage, 2004.

LERNER, R. M. et al. Positive youth development, participation in community youth development programs, and community contributions of fifth grade adolescents: Findings from the first wave of the 4-H study of positive youth development. Journal of Early Adolescence, n. 25, p. 17-71, 2005. Available from: < https://www.researchgate.net/publication/237237630_Positive_Youth_Development_Participation_in_Community_Youth_Development_Programs_and_Community_Contributions_of_Fifth-Grade_AdolescentsFindings_From_the_First_Wave_Of_the_4-H_Study_of_Positive_Youth_D $>$. Cited: 21 aug. 2018.

MACHELL, K.A.; DISABATO, D.J.; KASHDAN, T.B. Buffering the Negative Impact of Poverty on Youth: The Power of Purpose in Life. Social Indicators Research, v. 126, p. 845-861, 2016.

MALIN, H.; LIAUW, I.; DAMON, W. J. Purpose and Character Development in Early Adolescence. Youth Adolescence, v. 46, p. 1200-1215, 2017.

MARIANO, J. M.; SAVAGE, J. Exploring the language of youth purpose: references to positive states and coping styles by adolescents with different kinds of purpose. Journal of Research in Character Education, v. 7, n. 1, p. 1-24, 2009.

MARIANO, J. M. The psychological requirements of inclusion: a positive youth development view. In: JONES, P.; CARR, J.; FAUSKE, J. (Ed.). Leading for inclusion. New York, NY: Teacher's College Press, 2011.

MARIANO, J. M.; GOING, J. Youth purpose and positive youth development. In: LERNER, R. M.; LERNER, J. V.; BENSON, J. B. (Ed.). Advances in Child Development and Behavior, 2011. n. 41, p. 39-68.

MINEHAN, J. A.; NEWCOMB, M. D.; GALAIF, E. R. Predictors of adolescent drug use: Cognitive abilities, coping strategies and purpose in life. Journal of Child \& Adolescent Substance Abuse, v. 10, n. 2, p. 33-52, 2000.

PIAGET, J. Seis estudos de psicologia. 24th ed. (M. A. M. D'Amorim \& P. S. L Silva, Trans.). Rio de Janeiro: Forense Universitária, 2007.

PIAGET, J.; INHELDER, B. Da lógica da criança à lógica do adolescente. (D.M. Leite, Trans.). Rio de Janeiro: Forense Universitária, 1976. 
RAMEY H. L.; KRASNOR L. R.; BUSSERI M. A; GADBOIS S.; BOWKER A.; FINDLAY L. Measuring psychological engagement in youth activity involvement. Journal of Adolescence, v. 45, p. 237-249, 2015.

SHOSHANI, A.; RUSSO-NETZER, P. Exploring and assessing meaning in life in elementary school children: Development and validation of the meaning in life in children questionnaire (MIL-CQ). Personality and Individual Differences, v.104. p. 460-465, 2017.

SINK, C. A.; BULTSMA, S. A. Psychometric Analysis of the Life Perspectives Inventory and Implications for Assessing Characteristics of Adolescent Spirituality. Measurement \& Evaluation in Counseling \& Development, [s. 1.], v. 47, n. 2, p. 150-167, 2014.

STODDARD, S.A.; PIERCE, J. Promoting Positive Future Expectations During Adolescence: The Role of Assets. Am J Community Psychol, v. 56, p. 332-341, 2015. 